\title{
IN SILICO MINING FOR ALKALINE ENZYMES FROM METAGENOMIC DNA DATA OF GUT MICROBES OF THE LOWER TERMITE Coptotermes gestroi IN VIETNAM
}

\author{
Nguyen Minh Giang ${ }^{1}$, Do Thi Huyen ${ }^{2}$, Truong Nam Hai ${ }^{2}$ \\ ${ }^{1}$ Ho Chi Minh University of Pedagogy \\ ${ }^{2}$ Institute of Biotechnology, Vietnam Academy of Science and Techology
}

\begin{abstract}
ASBTRACT: The high alkaline proteases, lipases, cellulases and hemicellulases are important enzymes in research and industries. In this study, using the Alcapred software, the metagenomic DNA sequences of the gut flora of Coptotermes gestroi were analyzed to identify the enzymes that were specifically adapted to alkaline condition. The results show that 737 of 943 ORFs (accounting for $72 \%$ ) encoded proteases, 154 of 214 ORFs (holding 78\%) encoded lipases and 338 of 575 ORFs (accounting for 59\%) encoded cellulase and hemicelluase. All those enzymes were predicted to be alkaline enzymes. This study provide an overview picture of the alkaline enzyme groups of the gut flora of $C$. gestroi, and provide a good database for mining, isolation of the genes to produce recombinant enzymes.
\end{abstract}

Keywords: Coptotermes gestroi, alkaline enzyme, cellulase, gut, hemicellulase, lipase, metagenome, protease.

Citation: Nguyen Minh Giang, Do Thi Huyen, Truong Nam Hai, 2016. In silico mining for alkaline enzymes from metagenomic dna data of gut microbes of the lower termite Coptotermes gestroi in Vietnam. Tap chi Sinh hoc, 38(3): 374-383. DOI: 10.15625/0866-7160/v38n3.7811.

*Corresponding author: gdthgiang@gmail.com.

\section{INTRODUCTION}

Termites contribute substantially to the turnover of carbon and nitrogen in tropical ecosystems. Their diet consists exclusively of lignocellulose of various stages of decomposition, ranging from sound wood to humus. The digestion of this recalcitrant diet relies on the metabolic activities of a dense and diverse intestinal microbiota. In the gut of many lower termite Zootermopsis nevadensis, Reticulitermes lucifugus and $R$. flvipes, the $\mathrm{pH}$ was neutral to slightly acidic throughout, ranging from 5.5 to 7.5 . In many higher termites, the hindgut is compartmentalized to form several consecutive microbial bioreactors [1], and the $\mathrm{pH}$ of the special anterior hindgut is highly alkaline. In soil-feeding Termitinae such as Nasutitermes nigriceps and $N$. corniger, the $\mathrm{pH}$ increases sharply at the mixed segment and reaches its maximum $\mathrm{pH}$ of 12 in hindgut [3].

Since the hindgut of some termites are extremely alkaline $\mathrm{pH}$, we assumed that termite gut is a highly convenient mini ecosystem to exploit the alkaline enzymes of the intestinal microbiota. Therefore, we conducted a survey for alkaline enzymes such as proteases, lipases, cellulases and hemicellulases in the DNA sequences metagenome of microbiota in the gut of $C$. gestroi. If we could find many novel enzymes, they might be interested in both academic and industrial aspects.

\section{MATERIALS AND METHODS}

Genomic DNA was extracted from gut flora of $C$. gestroi extracted from the free-living microorganism in gut of $C$. gestroi collected from wood-nesting colonies in Hanoi and Hung Yen province in Vietnam according to the method described by Sambrook et al. [21] and sequenced using HiSeq2000 sequencing system (Illumina, San Diego, USA). Metagenomic DNA sequence data was analyzed using standard bioinformatics approach. A range of bioinformatic softwares such as BLAST, MEGAN, SOAP and the data in NCBI, KEGG, eggNOG were used to identify and account for ORFs [6]. 
Alkaline and acidic enzymes analysis: Based on the predicted ORFs annotated using KEGG and eggNOG, we used a sequence-based tool to discriminate acidic and alkaline enzymes. A feature selection technique was used to pick out a number of informative features. Based on these features, the support vector machine (SVM) analysis was performed to establish a prediction model. Prediction results demonstrate that the proposed method is reliable. Then, a free online database called AcalPred was built to provide a useful tool for basic academic study and industrial application of acidic and alkaline enzymes [12]. An overall accuracy of $96 \%$ was achieved, demonstrating that the proposed model is a powerful tool for the study on the adaptation of enzymes to acidic or alkaline environment.

\section{RESULTS AND DISCUSSION}

We present the metagenomic analysis of a large data set $(5.4 \mathrm{~Gb})$ generated by Illuminabased de novo sequencing of genomic DNA of the gut flora of lower termite, C. gestroi. To our best our knowledge, this study is the first successful application of high-throughput sequencing for the investigation of the gut flora of the lower termite, C. gestroi.

Metagenomic sequence analysis of the genomic DNA $(8.5 \mathrm{mg})$ extracted from the gut flora of C. gestroi Illumina platform yielded 5.6 $\mathrm{Gb}$ of sequence reads. Meta Gene Annotator [19] identified 125,431 putative ORFs. The functional profile of the metagenome of the flora was determined by the classification of predicted genes based on the eggNOG [20] and KEGG [16] databases. We found that the metagenomic sequences were distributed among typically prokaryotic eggNOG functional categories [20]. Among 125,431 ORFs, 36,477 ORFs were classified into enzyme families and 65,536 ORFs were predicted to be functional. The functional properties were determined using deeper levels of the eggNOG and KEGG classes [16, 20].

\section{Putative alkaline enzymes in the gut flora of C. gestroi termite}

We predicted alkaline and acidic enzymes such as proteases, lipases, cellulases and hemicellulases using Alcapred software. Among 125,341 ORFs, 943 were annotated to encode proteases, 214 encode lipases and 579 encode cellulases and hemicellulases (table 1).

Table 1. The total predicted ORF, protease, lipase, cellulase and hemicellulase of metagenome of the termite gut microbiota

\begin{tabular}{ccccc}
\hline Enzymes & Total ORF & Proteases & Lipases & $\begin{array}{c}\text { Cellulases and } \\
\text { hemicellulases }\end{array}$ \\
\hline Number of sequences & 125341 & 943 & 214 & 578 \\
\hline
\end{tabular}

The number of proteinases sequences (934) was the highest. This is reasonable because proteases are present in all microorganisms in the gut, even flagellate protozoan symbionts [22]. Proteases are critically important in a diverse biological processes including the regulation of the metabolism of cells and are essential constituents of all forms of life on earth [23]. So it is reasonable that protease is the largest enzyme group in cells compared with other enzymes.

In the gut of low and high termites, the most outstanding microbe in the community is bacteria. They dominate not only on the intestinal wall of termite gut but also symbiotic flagellate or protozoa [26]. Metagenomic DNA sequence analysis of the gut flora of $C$. gestroi revealed that $80 \%$ of the total ORFs are belong to bacteria.

Lignocellulose is main constituent of termite food with high percentage of cellulose and hemicellulose. Trend is emerging suggesting collaboration among termite-derived genes expressed in the salivary gland/foregut, midgut and symbiont genes expressed in the hindgut. However, many studies demonstrated that cellulolytic and hemicellulolytic enzymes from microorganism in termite gut is quiet rich 
[11] such as: $73 \%$ cellulases and xylanases were known in Microcerotermes sp. [27]; the series of cellulase in the foregut/salivary gland; and a rich diversity of derived GHFs in the hindgut. Of the various exoglucanases are undeniably the most diverse [23]. In our results, we identified 578 cellulases and hemicellulases sequences, which is just behind the number of proteases and much higher than the number of lipase sequences (214)

\section{Putative alkaline protease and lipase}

For the sequnce data encoding different enzyme groups, we used the Alcapred software to predict proteases and lipases that are tolerant to alkaline condition (table 2).

As shown in table 2, $737(78 \%)$ out of 943 proteinase ORFs are alkaline proteases and $154(72 \%)$ of 214 lipase ORFs are alkaline lipases.

Table 2. Summary of predicted alkaline and acidic protease and lipase in the gut of $C$. gestroi

\begin{tabular}{cccccc}
\hline Enzymes & $\begin{array}{c}\text { Total } \\
\text { sequences }\end{array}$ & $\begin{array}{c}\text { Alkaline } \\
\text { sequences }\end{array}$ & Percentage (\%) & $\begin{array}{c}\text { Acidic } \\
\text { sequences }\end{array}$ & Percentage \\
\hline Protease & 943 & 737 & $78 \%$ & 206 & $22 \%$ \\
Lipase & 214 & 154 & $72 \%$ & 60 & $28 \%$ \\
\hline
\end{tabular}

The hindgut of termites is the anaerobic fermentation tank containing a variety of different microorganisms having various enzymes that tolerate alkaline environment. Origin and distribution of proteases have been studied in detail in higher termites [REF]. However, little information is available about proteases in lower termites [24]. Only few publications about the proportion of alkaline proteases and lipases in the gut flora of the termites. Alkaline proteases and alkaline lipases were found originated from higher termite, Nasutitermes corniger and its enteric flora [10] . Our present results give the overall clarification of the high alkaline proteases and lipases of the gut flora of lower termite, C. gestroi. Among the ORFs encoding alkaline enzymes, 175 of 737 alkaline proteases ORFs and 33 of 154 alkaline lipases ORFs have alkaline index of higher than 0.99 (table 3, 4).

Table 3. Summary of the gene sequences encoding proteases having alkaline index of 0.99

\begin{tabular}{lcccc}
\hline STT & Code gene & Alkaline index & Acidic index & Protease \\
\hline 1 & GL0054846 & 1.000000 & 0.000000 & regulator of sigma E protease \\
2 & GL0054872 & 1.000000 & 0.000000 & carboxyl-terminal processing protease \\
3 & GL0054905 & 1.000000 & 0.000000 & ATP-dependent Lon protease \\
4 & GL0054967 & 1.000000 & 0.000000 & putative protease \\
5 & GL0055331 & 1.000000 & 0.000000 & Lon-like ATP-dependent protease \\
6 & GL0056476 & 1.000000 & 0.000000 & regulator of sigma E protease \\
7 & GL0056477 & 1.000000 & 0.000000 & ATP-dependent Lon protease \\
8 & GL0057752 & 1.000000 & 0.000000 & ATP-dependent Lon protease \\
9 & GL0057900 & 1.000000 & 0.000000 & zinc protease \\
10 & GL0058152 & 1.000000 & 0.000000 & cell wall-associated protease \\
11 & GL0058203 & 1.000000 & 0.000000 & major intracellular serine protease \\
12 & GL0058234 & 1.000000 & 0.000000 & hydrogenase 1 maturation protease \\
13 & GL0058638 & 1.000000 & 0.000000 & ATP-dependent Lon protease \\
14 & GL0058989 & 1.000000 & 0.000000 & carboxyl-terminal processing protease \\
15 & GL0059141 & 1.000000 & 0.000000 & cell wall-associated protease \\
16 & GL0059540 & 1.000000 & 0.000000 & ATP-dependent Lon protease \\
17 & GL0059860 & 1.000000 & 0.000000 & ATP-dependent Lon protease \\
18 & GL0060204 & 1.000000 & 0.000000 & Lon-like ATP-dependent protease \\
19 & GL0060412 & 1.000000 & 0.000000 & carboxyl-terminal processing protease \\
20 & GL0060449 & 1.000000 & 0.000000 & regulator of sigma E protease \\
\hline
\end{tabular}


Nguyen Minh Giang et al.

\begin{tabular}{|c|c|c|c|c|}
\hline 21 & GL0060495 & 1.000000 & 0.000000 & zinc protease \\
\hline 22 & GL0060843 & 1.000000 & 0.000000 & zinc protease \\
\hline 23 & GL0060923 & 1.000000 & 0.000000 & ATP-dependent Lon protease \\
\hline 24 & GL0061012 & 1.000000 & 0.000000 & ATP-dependent Lon protease \\
\hline 25 & GL0061226 & 1.000000 & 0.000000 & carboxyl-terminal processing protease \\
\hline 26 & GL0061851 & 1.000000 & 0.000000 & carboxyl-terminal processing protease \\
\hline 27 & GL0061932 & 1.000000 & 0.000000 & ATP-dependent Lon protease \\
\hline 28 & GL0005083 & 1.000000 & 0.000000 & ATP-dependent Lon protease \\
\hline 29 & GL0062064 & 1.000000 & 0.000000 & ATP-dependent Lon protease \\
\hline 30 & GL0062383 & 1.000000 & 0.000000 & carboxyl-terminal processing protease \\
\hline 31 & GL0062512 & 1.000000 & 0.000000 & zinc protease \\
\hline 32 & GL0063609 & 1.000000 & 0.000000 & putative metalloprotease \\
\hline 33 & GL0063636 & 1.000000 & 0.000000 & zinc protease \\
\hline 34 & GL0064020 & 1.000000 & 0.000000 & carboxyl-terminal processing protease \\
\hline 35 & GL0064465 & 1.000000 & 0.000000 & zinc protease \\
\hline 36 & GL0064477 & 1.000000 & 0.000000 & ATP-dependent Lon protease \\
\hline 37 & GL0064544 & 1.000000 & 0.000000 & zinc protease \\
\hline 38 & GL0064904 & 1.000000 & 0.000000 & regulator of sigma $\mathrm{E}$ protease \\
\hline 39 & GL0065106 & 1.000000 & 0.000000 & putative metalloprotease \\
\hline 40 & GL0065431 & 1.000000 & 0.000000 & tricorn protease \\
\hline 41 & GL0066206 & 1.000000 & 0.000000 & ATP-dependent Lon protease \\
\hline 42 & GL0067180 & 1.000000 & 0.000000 & carboxyl-terminal processing protease \\
\hline 43 & GL0067487 & 1.000000 & 0.000000 & tricorn protease \\
\hline 44 & GL0068015 & 1.000000 & 0.000000 & carboxyl-terminal processing protease \\
\hline 45 & GL0068234 & 1.000000 & 0.000000 & putative protease \\
\hline 46 & GL0068607 & 1.000000 & 0.000000 & carboxyl-terminal processing protease \\
\hline 47 & GL0069912 & 1.000000 & 0.000000 & regulator of sigma $E$ protease \\
\hline 48 & GL0070217 & 1.000000 & 0.000000 & regulator of sigma $\mathrm{E}$ protease \\
\hline 49 & GL0070881 & 1.000000 & 0.000000 & carboxyl-terminal processing protease \\
\hline 50 & GL0070891 & 1.000000 & 0.000000 & ATP-dependent Lon protease \\
\hline 51 & GL0071299 & 1.000000 & 0.000000 & carboxyl-terminal processing protease \\
\hline 52 & GL0071667 & 1.000000 & 0.000000 & ATP-dependent Lon protease \\
\hline 53 & GL0071998 & 1.000000 & 0.000000 & ATP-dependent Lon protease \\
\hline 54 & GL0072105 & 1.000000 & 0.000000 & putative protease \\
\hline 55 & GL0072492 & 1.000000 & 0.000000 & putative protease \\
\hline 56 & GL0072552 & 1.000000 & 0.000000 & regulator of sigma $\mathrm{E}$ protease \\
\hline 57 & GL0072785 & 1.000000 & 0.000000 & zinc protease \\
\hline 58 & GL0072957 & 1.000000 & 0.000000 & carboxyl-terminal processing protease \\
\hline 59 & GL0073275 & 1.000000 & 0.000000 & putative protease \\
\hline 60 & GL0073638 & 1.000000 & 0.000000 & carboxyl-terminal processing protease \\
\hline 61 & GL0073667 & 1.000000 & 0.000000 & Lon-like ATP-dependent protease \\
\hline 62 & GL0074179 & 1.000000 & 0.000000 & tricorn protease \\
\hline 63 & GL0074314 & 1.000000 & 0.000000 & carboxyl-terminal processing protease \\
\hline 64 & GL0074377 & 1.000000 & 0.000000 & ATP-dependent Lon protease \\
\hline 65 & GL0074576 & 1.000000 & 0.000000 & carboxyl-terminal processing protease \\
\hline 66 & GL0074589 & 1.000000 & 0.000000 & subtilase-type serine protease \\
\hline 67 & GL0075722 & 1.000000 & 0.000000 & ATP-dependent Lon protease \\
\hline 68 & GL0076003 & 0.999999 & 0.000001 & ATP-dependent Lon protease \\
\hline 69 & GL0076249 & 0.999999 & 0.000001 & carboxyl-terminal processing protease \\
\hline 70 & GL0077278 & 0.999999 & 0.000001 & cell wall-associated protease \\
\hline 71 & GL0077302 & 0.999999 & 0.000001 & ATP-dependent Lon protease \\
\hline 72 & GL0077595 & 0.999999 & 0.000001 & ATP-dependent Lon protease \\
\hline 73 & GL0078352 & 0.999999 & 0.000001 & carboxyl-terminal processing protease \\
\hline 74 & GL0078771 & 0.999999 & 0.000001 & ATP-dependent Lon protease \\
\hline 75 & GL0079155 & 0.999999 & 0.000001 & carboxyl-terminal processing protease \\
\hline
\end{tabular}


In silico mining for alkaline enzymes

\begin{tabular}{|c|c|c|c|c|}
\hline 76 & GL0079245 & 0.999999 & 0.000001 & ATP-dependent Lon protease \\
\hline 77 & GL0080059 & 0.999999 & 0.000001 & zinc protease \\
\hline 78 & GL0080468 & 0.999999 & 0.000001 & Lon-like ATP-dependent protease \\
\hline 79 & GL0080662 & 0.999999 & 0.000001 & carboxyl-terminal processing protease \\
\hline 80 & GL0080682 & 0.999999 & 0.000001 & carboxyl-terminal processing protease \\
\hline 81 & GL0081284 & 0.999999 & 0.000001 & regulator of sigma $\mathrm{E}$ protease \\
\hline 82 & GL0081653 & 0.999999 & 0.000001 & zinc protease \\
\hline 83 & GL0081869 & 0.999999 & 0.000001 & putative protease \\
\hline 84 & GL0082183 & 0.999999 & 0.000001 & subtilase-type serine protease \\
\hline 85 & GL0082905 & 0.999998 & 0.000002 & zinc protease \\
\hline 86 & GL0082905 & 0.999998 & 0.000002 & zinc protease \\
\hline 87 & GL0006359 & 0.999998 & 0.000002 & carboxyl-terminal processing protease \\
\hline 88 & GL0006895 & 0.999998 & 0.000002 & putative protease \\
\hline 89 & GL0007102 & 0.999998 & 0.000002 & putative protease \\
\hline 90 & GL0085754 & 0.999998 & 0.000002 & carboxyl-terminal processing protease \\
\hline 91 & GL0085819 & 0.999998 & 0.000002 & regulator of sigma $\mathrm{E}$ protease \\
\hline 92 & GL0086188 & 0.999998 & 0.000002 & putative protease \\
\hline 93 & GL0086783 & 0.999998 & 0.000002 & tricorn protease \\
\hline 94 & GL0087433 & 0.999998 & 0.000002 & zinc protease \\
\hline 95 & GL0087476 & 0.999998 & 0.000002 & putative protease \\
\hline 96 & GL0088709 & 0.999998 & 0.000002 & Lon-like ATP-dependent protease \\
\hline 97 & GL0089124 & 0.999998 & 0.000002 & hydrogenase 3 maturation protease \\
\hline 98 & GL0089539 & 0.999998 & 0.000002 & ATP-dependent Lon protease \\
\hline 99 & GL0089692 & 0.999997 & 0.000003 & regulator of sigma $\mathrm{E}$ protease \\
\hline 100 & GL0090100 & 0.999997 & 0.000003 & subtilase-type serine protease \\
\hline 101 & GL0090101 & 0.999997 & 0.000003 & subtilase-type serine protease \\
\hline 102 & GL0090767 & 0.999997 & 0.000003 & tricorn protease \\
\hline 103 & GL0091105 & 0.999997 & 0.000003 & putative protease \\
\hline 104 & GL0091196 & 0.999997 & 0.000003 & regulator of sigma $\mathrm{E}$ protease \\
\hline 105 & GL0091332 & 0.999997 & 0.000003 & ATP-dependent Lon protease \\
\hline 106 & GL0092578 & 0.999997 & 0.000003 & putative protease \\
\hline 107 & GL0093355 & 0.999997 & 0.000003 & ATP-dependent Lon protease \\
\hline 108 & GL0093891 & 0.999997 & 0.000003 & carboxyl-terminal processing protease \\
\hline 109 & GL0094050 & 0.999997 & 0.000003 & Lon-like ATP-dependent protease \\
\hline 110 & GL0094306 & 0.999996 & 0.000004 & putative protease \\
\hline 111 & GL0094337 & 0.999996 & 0.000004 & ATP-dependent Lon protease \\
\hline 112 & GL0094379 & 0.999996 & 0.000004 & spore protease \\
\hline 113 & GL0094531 & 0.999996 & 0.000004 & putative protease \\
\hline 114 & GL0094533 & 0.999996 & 0.000004 & putative protease \\
\hline 115 & GL0095247 & 0.999996 & 0.000004 & hydrogenase 3 maturation protease \\
\hline 116 & GL0095457 & 0.999995 & 0.000005 & Lon-like ATP-dependent protease \\
\hline 117 & GL0096224 & 0.999995 & 0.000005 & regulator of sigma $\mathrm{E}$ protease \\
\hline 118 & GL0096533 & 0.999995 & 0.000005 & regulator of sigma $\mathrm{E}$ protease \\
\hline 119 & GL0097138 & 0.999995 & 0.000005 & putative protease \\
\hline 120 & GL0097401 & 0.999995 & 0.000005 & putative protease \\
\hline 121 & GL0097824 & 0.999995 & 0.000005 & putative protease \\
\hline 122 & GL0098212 & 0.999994 & 0.000006 & carboxyl-terminal processing protease \\
\hline 123 & GL0098352 & 0.999994 & 0.000006 & putative protease \\
\hline 124 & GL0098435 & 0.999994 & 0.000006 & zinc protease \\
\hline 125 & GL0099896 & 0.999994 & 0.000006 & hydrogenase 3 maturation protease \\
\hline 126 & GL0100379 & 0.999994 & 0.000006 & ATP-dependent Lon protease \\
\hline 127 & GL0100766 & 0.999994 & 0.000006 & ATP-dependent Lon protease \\
\hline 128 & GL0100872 & 0.999994 & 0.000006 & putative protease \\
\hline 129 & GL0100931 & 0.999993 & 0.000007 & ATP-dependent Lon protease \\
\hline 130 & GL0101204 & 0.999993 & 0.000007 & ATP-dependent Lon protease \\
\hline
\end{tabular}


Nguyen Minh Giang et al.

\begin{tabular}{|c|c|c|c|c|}
\hline 131 & GL0101716 & 0.999993 & 0.000007 & ATP-dependent Lon protease \\
\hline 132 & GL0101933 & 0.999993 & 0.000007 & putative metalloprotease \\
\hline 133 & GL0102081 & 0.999993 & 0.000007 & regulator of sigma $\mathrm{E}$ protease \\
\hline 134 & GL0102493 & 0.999992 & 0.000008 & ATP-dependent Lon protease \\
\hline 135 & GL0102586 & 0.999992 & 0.000008 & carboxyl-terminal processing protease \\
\hline 136 & GL0102946 & 0.999991 & 0.000010 & carboxyl-terminal processing protease \\
\hline 137 & GL0103635 & 0.999991 & 0.000010 & zinc protease \\
\hline 138 & GL0103640 & 0.999990 & 0.000010 & putative protease \\
\hline 139 & GL0103741 & 0.999990 & 0.000010 & ATP-dependent Lon protease \\
\hline 140 & GL0104021 & 0.999989 & 0.000011 & ATP-dependent Lon protease \\
\hline 141 & GL0008440 & 0.999989 & 0.000011 & Lon-like ATP-dependent protease \\
\hline 142 & GL0104067 & 0.999989 & 0.000011 & muramoyltetrapeptide carboxypeptidase \\
\hline 143 & GL0104382 & 0.999988 & 0.000012 & D-alanyl-D-alanine carboxypeptidase \\
\hline 144 & GL0105276 & 0.999988 & 0.000012 & aminoacylhistidine dipeptidase \\
\hline 145 & GL0105590 & 0.999987 & 0.000013 & tripeptide aminopeptidase \\
\hline 146 & GL0105694 & 0.999987 & 0.000013 & aminoacylhistidine dipeptidase \\
\hline 147 & GL0105744 & 0.999987 & 0.000013 & putative endopeptidase \\
\hline 148 & GL0106295 & 0.999986 & 0.000014 & Aminopeptidase \\
\hline 149 & GL0107933 & 0.999986 & 0.000014 & acylaminoacyl-peptidase \\
\hline 150 & GL0108253 & 0.999986 & 0.000014 & putative endopeptidase \\
\hline 151 & GL0108449 & 0.999985 & 0.000015 & Aminopeptidase \\
\hline 152 & GL0108581 & 0.999985 & 0.000015 & Aminopeptidase \\
\hline 153 & GL0109350 & 0.999985 & 0.000015 & putative endopeptidase \\
\hline 154 & GL0109963 & 0.999984 & 0.000016 & X-Pro aminopeptidase \\
\hline 155 & GL0110057 & 0.999983 & 0.000017 & X-Pro dipeptidase \\
\hline 156 & GL0110079 & 0.999983 & 0.000017 & IgA-specific serine endopeptidase \\
\hline 157 & GL0110198 & 0.999982 & 0.000018 & X-Pro dipeptidase \\
\hline 158 & GL0110319 & 0.997478 & 0.002522 & gamma-glutamyltranspeptidase \\
\hline 159 & GL0110421 & 0.997472 & 0.002528 & D-aminopeptidase \\
\hline 160 & GL0110658 & 0.997459 & 0.002541 & D-alanyl-D-alanine carboxypeptidase \\
\hline 161 & GL0110664 & 0.997457 & 0.002543 & leucyl aminopeptidase \\
\hline 162 & GL0110690 & 0.997444 & 0.002556 & prolyl oligopeptidase \\
\hline 163 & GL0110743 & 0.997443 & 0.002557 & Aminopeptidase \\
\hline 164 & GL0110837 & 0.997418 & 0.002582 & methionyl aminopeptidase \\
\hline 165 & GL0110963 & 0.997417 & 0.002583 & X-Pro aminopeptidase \\
\hline 166 & GL0111249 & 0.997389 & 0.002611 & O-sialoglycoprotein endopeptidase \\
\hline 167 & GL0111426 & 0.997349 & 0.002651 & tripeptide aminopeptidase \\
\hline 168 & GL0111488 & 0.997329 & 0.002671 & D-alanyl-D-alanine carboxypeptidase \\
\hline 169 & GL0111629 & 0.997233 & 0.002767 & Aminopeptidase \\
\hline 170 & GL0111698 & 0.997222 & 0.002778 & putative endopeptidase \\
\hline 171 & GL0111759 & 0.997181 & 0.002819 & glutamyl endopeptidase \\
\hline 172 & GL0112043 & 0.997118 & 0.002882 & Aminopeptidase \\
\hline 173 & GL0112548 & 0.997098 & 0.002902 & O-sialoglycoprotein endopeptidase \\
\hline 174 & GL0112732 & 0.997001 & 0.002999 & X-Pro aminopeptidase \\
\hline 175 & GL0113031 & 0.996930 & 0.003070 & X-Pro aminopeptidase \\
\hline
\end{tabular}

Table 4. Summary of the gene sequences encoding lipases having alkaline index of 0.99

\begin{tabular}{lcccc}
\hline STT & Code gene & Alkaline index & Acidic index & Enzyme lipase \\
\hline 1 & GL0094408 & 1.000000 & 0.000000 & esterase / lipase \\
2 & GL0095714 & 1.000000 & 0.000000 & triacylglycerol lipase \\
3 & GL0098504 & 1.000000 & 0.000000 & triacylglycerol lipase \\
4 & GL0100660 & 1.000000 & 0.000000 & Lysophospholipase \\
5 & GL0102502 & 1.000000 & 0.000000 & triacylglycerol lipase \\
\hline
\end{tabular}


In silico mining for alkaline enzymes

\begin{tabular}{|c|c|c|c|c|}
\hline 6 & GL0103848 & 1.000000 & 0.000000 & esterase / lipase \\
\hline 7 & GL0115777 & 1.000000 & 0.000000 & phospholipase A1 \\
\hline 8 & GL0028122 & 1.000000 & 0.000000 & phospholipase A1 \\
\hline 9 & GL0052713 & 1.000000 & 0.000000 & phospholipase A1 \\
\hline 10 & GL0057522 & 1.000000 & 0.000000 & phospholipase A1 \\
\hline 11 & GL0091897 & 1.000000 & 0.000000 & phospholipase A1 \\
\hline 12 & GL0097086 & 1.000000 & 0.000000 & phospholipase A1 \\
\hline 13 & GL0102371 & 1.000000 & 0.000000 & phospholipase A1 \\
\hline 14 & GL0102961 & 1.000000 & 0.000000 & phospholipase A1 \\
\hline 15 & GL0130369 & 1.000000 & 0.000000 & phospholipase C \\
\hline 16 & GL0019568 & 1.000000 & 0.000000 & phospholipase C \\
\hline 17 & GL0092972 & 1.000000 & 0.000000 & phospholipase C \\
\hline 18 & GL0113097 & 1.000000 & 0.000000 & phospholipase C \\
\hline 19 & GL0017374 & 0.999999 & 0.000001 & phospholipase D \\
\hline 20 & GL0018116 & 0.999999 & 0.000001 & phospholipase D \\
\hline 21 & GL0033413 & 0.999999 & 0.000001 & phospholipase D \\
\hline 22 & GL0056310 & 0.999999 & 0.000001 & phospholipase D \\
\hline 23 & GL0071465 & 0.999999 & 0.000001 & phospholipase D \\
\hline 24 & GL0071465 & 0.999999 & 0.000001 & phospholipase D \\
\hline 25 & GL0076794 & 0.999998 & 0.000002 & phospholipase D \\
\hline 26 & GL0082514 & 0.999996 & 0.000004 & phospholipase D \\
\hline 27 & GL0087982 & 0.999996 & 0.000004 & phospholipase D \\
\hline 28 & GL0008869 & 0.999994 & 0.000006 & phospholipase D \\
\hline 29 & GL0104048 & 0.999993 & 0.000007 & phospholipase A1 \\
\hline 30 & GL0108498 & 0.999991 & 0.000009 & phospholipase A1 \\
\hline 31 & GL0108499 & 0.999991 & 0.000009 & phospholipase A1 \\
\hline 32 & GL0108547 & 0.999991 & 0.000009 & phospholipase A1 \\
\hline 33 & GL0113097 & 0.999985 & 0.000015 & phospholipase D \\
\hline
\end{tabular}

Table 5. Summary of predicted alkaline and acidic cellulases and hemicellulases in the gut flora of C. gestroi

\begin{tabular}{cccccc}
\hline Enzymes & $\begin{array}{c}\text { Total } \\
\text { sequences }\end{array}$ & $\begin{array}{c}\text { Alkaline } \\
\text { sequences }\end{array}$ & $\begin{array}{c}\text { Percentage } \\
(\%)\end{array}$ & $\begin{array}{c}\text { Acidic } \\
\text { sequences }\end{array}$ & $\begin{array}{c}\text { Percentage } \\
(\%)\end{array}$ \\
\hline $\begin{array}{c}\text { Cellulases and } \\
\text { hemicellulases }\end{array}$ & 578 & 338 & $59 \%$ & 240 & $41 \%$ \\
\hline
\end{tabular}

Putative alkaline and acidic cellulases and hemicellulases

Among a total of 575 cellulolytic and hemicellulolytic enzymes, 338 (59\%) were predicted to be alkaline enzymes. The number of alkaline cellulases and hemicellulases are lower than those of alkaline proteases and lipases,.

It is already well-known that cellulases and hemicellulases are abundant in the symbiotic organisms in the gut of termites to degrade cellulose and hemicellulose [14, 17]. Diversity and of lignocellulose-degrading alkaline enzymes and their function in the termite gut microbial community have been reported [17]. Cellulases and hemicellulases from the gut flora of termites such as $R$. flavipes, $R$. speratus and Macrotermes subhyalinus have optimal $\mathrm{pH}$ around 5-7, and those from Microcerotermes sp. have wider optimal $\mathrm{pH}$ range of 5.0-10.0. In case of cellulolytic and hemmicellulolytic enzymes of Sarocladium kiliense and Trichoderma virens isolated from the gut of the lower termite, $R$. santonensis, optimal $\mathrm{pH}$ range was $\mathrm{pH} 9-10$ [27]. In the higher termite, 
Nguyen Minh Giang et al.

Nasutitermes corniger, $\mathrm{pH}$ of the gut reaches as high as 11 [10]. In this study, there is not much difference between the proportion of the predicted alkaline (59\%) and acidic (41\%) cellulases and hemicellulases of the gut flora of C. gestroi In contrast, in cases of proteinases and lipases, the proportion of alkaline enzymes was much higher than acidic ones. Presence of huge number of alkaline enzymes in the gut flora suggest that those microbiota are suitable to suvive alkaline environment of the gut of C. gestroi. Extracellular enzymes produced/ released from such microbiota are likely to have their optimum $\mathrm{pH}$ of alkaline range [7].

In this study we are interested in the ability of the cellulase and hemicellulase enzymes that can resist alkaline environment. Using Alcapred software, we found that the majority of alkaline cellulases and hemicellulases have very high alkaline index; 41 alkaline cellulases sequences and 40 alkaline hemicellulases have alkaline index of $>0.99$. All of them belong to betaglucosidase and alpha- $\mathrm{N}$-arabinofuranosidase.

\section{CONCLUSION}

Using Alcapred software, high percentages of proteases, lipases, cellulases and hemicellulases of the gut flora of $C$. gestroi were predicted as alkaline enzymes. These results might be useful for the effective utilization of novel alkaline enzymes in the industries. This is the first prediction of the alkaline enzyme groups of the gut flora of $C$. gestroi termites. The results of this study provide a comprehensive picture of alkaline tolerance of various enzyme groups which has not been reported previously.

Acknowledgments: This research was supported by the Cooperation Project "Isolation of genes encoding lignocellulolytic enzymes from Vietnam termite gut microflora by Metagenomic approach" from the Ministry of Science and Technology, Vietnam, and implementad at the National Key Laboratory of Gene Technology, Institute of Biotechnology, VAST, Vietnam, and the Applied Bacteriology Laboratory, National Food Research Institute, National Agriculture and Food Research Organization (NARO), Japan.

\section{REFERENCES}

1. Bignell D. E., Eggleton P., 1995. On the elevated intestinal $\mathrm{pH}$ of higher termites (Isoptera: Termitidae). Insect Soc., 42(1): 57-69.

2. Brune A., Kühl M., 1996. pH profiles of the extremely alkaline hindguts of soil-feeding termites (Isoptera: Termitidae) determined with microelectrodes. J. Insect Physiol., 42(11): 1121-1122.

3. Brune A., Emerson D., Breznak J. A., 1995. The termite gut microflora as an oxygen sink: microelectrode determination of oxygen and $\mathrm{pH}$ gradients in guts of lower and higher termites. Appl. Environ. Microbiol., 61(7): 2681-2687.

4. Chandrasekharaiah M., Thulasi A., Bagath M., Kumar D. P., Santosh S. S., Palanivel C., Jose V. L., Sampath K. T., 2011. Molecular cloning, expression and characterization of a novel feruloyl esterase enzyme from the symbionts of termite (Coptotermes formosanus) gut. BMB Rep., 44(1): 52-57.

5. Cherif S., Mnif S., Hadrich F., Abdelkafi S., Sayadi S., 2011. A newly high alkaline lipase: an ideal choice for application in detergent formulations. Lipids Health Dis. 10: 221 .

6. Do T. H., Nguyen T. T., Nguyen T. N., Le Q. G., Nguyen C., Kimura K., Truong N. H., 2014. Mining biomass-degrading genes through Illumina-based denovo sequencing and metagenomic analysis of free-living bacteria in the gut of the lower termite Coptotermes gestroi harvested in Vietnam. J Biosci Bioeng., 118(6): 665-671.

7. Gessesse A., Gashe B. A., 1997. Production of alkaline xylanase by alkaliphic Bacillus sp isolated alkaline soda lake. J. Appl. Microbiol., 83(4): 402-406.

8. Hongoh Y., Ohkuma M., Kudo T., 2003. Molecular analysis of bacterial microbiota in the gut of the termite Reticulitermes speratus (Isoptera: Rhinotermitidae). FEMS Microbiol. Ecol., 44(2): 231-242. 
9. Huson D. H., Auch A. F., Qi J., Schuster S. C., 2007. MEGAN analysis of metagenomic data, Genome Res., 17(3): 377-386.

10. Köhler T., Dietrich C., Scheffrahn RH., Brune A., 2012. High-resolution analysis of gut environment and bacterial microbiota reveals functional compartmentation of the gut in wood-feeding higher termites (Nasutitermes spp.). Appl. Environ. Microbiol., 78(13): 4691-4701.

11. Kudo T., 2009. Termite-microbe symbiotic system and its efficient degradation of lignocellulose. Biosci. Biotechnol. Biochem., 73(12): 2561-2567.

12. Lin H., Chen W., Ding H., 2013. AcalPred: A sequence-based tool for discriminating between acidic and alkaline enzymes. PLoS ONE 8(10): e75726.

13. Luo, R., Liu, B., Xie, Y., Li, Z., Huang, W., Yuan, J., He, G., Chen, Y., Pan, Q., Liu, Y., et al., 2012. SOAPdenovo2: an empirically improved memory-efficient short-read de novo assembler, GigaScience, 1(1): 18.

14. Mattéotti C., Thonart P., Francis F., Haubruge E., Destain J., Brasseur C., Bauwens J., De Pauw E., Portetelle D., Vandenbol M., 2011. New glucosidase activities identified by functional screening of a genomic DNA library from the gut microbiota of the termite Reticulitermes santonensis. Microbiol Res., 166(8): 62942.

15. Michael J., Liszka E., Schneider E., Clark D. S., 2012. Nature versus nurture: developing enzymes that function under extreme conditions. Annu. Rev. Chem. Biomol. Eng., 3: 77-102.

16. Mitra S., Rupek P., Richter D. C., Urich T., Gilbert J. A., Meyer F., Wilke A., Huson D. H., 2011. Functional analysis of metagenomes and metatranscriptomes using SEED and KEGG. BMC Bioinformatics. 12(Suppl 1): S21.

17. Ni J., Tokuda G., Takehara M., Watanabe H. 2007. Heterologous expression and enzymatic characterization of $\beta$-glucosidase from the drywood-eating termite, Neotermes koshunensis. Appl. Entomol. Zool., 42(3): 457-463.

18. Nimchua T., Thongaram T., Uengwetwanit T., Pongpattanakitshote S., Eurwilaichitr L., 2012. Metagenomic analysis of novel lignocellulose-degrading enzymes from higher termite guts inhabiting microbes. J. Microbiol. Biotechnol., 22(4): 462-9.

19. Noguchi H., Taniguchi T., Itoh T., 2008. MetaGeneAnnotator: detecting speciesspecific patterns of ribosomal binding site for precise gene prediction in anonymous prokaryotic and phage genomes. DNA Res., 15: 387-396.

20. Powell S., Szklarczyk D., Trachana K., Roth A., Kuhn M., Muller J., Arnold R., Rattei T., Letunic I., Doerks T., 2011. eggNOGv3.0: orthologous groups covering 1133 organisms at 41 different taxonomic ranges. Nucleic Acids Res., 40: D284-289.

21. Sambrook J., Russell D. W., 2008. Molecular cloning: a laboratory manual. Cold Spring Harbor Laboratory Press.

22. Scharf M. E., Karl Z. J., Sethi A., Boucias D. G., 2011. Multiple levels of synergistic collaboration in termite lignocellulose digestion, PLoS One. 6(7): e21709.

23. Scharf M. E., Tartar A., 2008. Termite digestomes as sources for novel lignocellulases. Biofuels, Bioprod. Bioref. DOI: $10.1002 / \mathrm{bbb} .107$.

24. Smith A., Scharf M. E., Roberto M., Philip G., Koehler G., 2009. pH Optimization of gut cellulase and xylanase activities from the Eastern subterranean termite, Reticulitermes flvipes (Isoptera: Rhinotermitidae). Sociobiol., 54: 199-210.

25. Sethi A., Xue Q. G., La Peyre J. F., Delatte J., Husseneder C., 2011. Dual origin of gut proteases in Formosan subterranean termites (Coptotermes formosanus Shiraki) (Isoptera: Rhinotermitidae). Comp Biochem Physiol A Mol Integr Physiol., 159(3): 261-267.

26. Szalanski A. L., Austin J. W., Scheffrahn R. H., Messenger M. T., 2004. Molecular diagnostics of the formosan subterranean 
termite (Isoptera: Rhinotermitidae). Fla. Entomol., 87: 145-151.

27. Tarayre C., Bauwens J., Brasseur C., Mattéotti C., Millet C., Guiot PA., Destain J., Vandenbol M., Portetelle D., De Pauw E., Haubruge E., Francis F., Thonart P., 2015. Isolation and cultivation of xylanolytic and cellulolytic Sarocladium kiliense and Trichoderma virens from the gut of the termite Reticulitermes santonensis. Environ Sci Pollut Res Int., 22(6): 4369-4382.

28. Tartar A., Wheeler M. M., Zhou X., Coy M. R., Boucias D. G., Scharf, M. E., 2009. Parallel metatranscriptome analyses of host and symbiont gene expression in the gut of the termite Reticulitermes flavipes. Biotechnol. Biofuels., 2: 25.

29. Watanabe H., Nakamura M., Tokuda G., Yamaoka I., Scrivener AM., Noda H., 1997.
30. Site of secretion and properties of endogenous endo-beta-1,4-glucanase components from Reticulitermes speratus (Kolbe), a Japanese subterranean termite. Insect Biochem Mol Biol., 27(4): 305-313.

31. Warnecke F., Luginbühl P., Ivanova N., Ghassemian M., Richardson T. H., Stege J. T., Cayouette M., McHardy A. C., Djordjevic G, Aboushadi N, Sorek R, Tringe S. G., Podar M., Martin H. G., Kunin V., Dalevi D., Madejska J., Kirton E., Platt D., Szeto E., Salamov A., Barry K., Mikhailova N., Kyrpides N. C., Matson E. G., Ottesen E. A., Zhang X., Hernández M., Murillo C., Acosta L. G., Rigoutsos I., Tamayo G., Green B. D., Chang C., Rubin E. M., Mathur E. J., Robertson D. E., Hugenholtz P., Leadbetter J. R., 2007. Metagenomic and functional analysis of hindgut microbiota of a wood-feeding higher termite. Nature, 450: 560-565.

\title{
SỬ DƯNG VI TÍNH KHAI THÁC CÁC ENZYME CHỊU KIỀM TỪ DŨ LIỆ DNA METAGENOME VI SINH VẠT SỐNG TRONG RUỘT MỐI BẬC THẤP coptotermes gestroi Ở VIỆT NAM
}

\author{
Nguyễn Minh Giang ${ }^{1}$, Đỗ Thị Huyền ${ }^{2}$, Trương Nam Hải ${ }^{2}$ \\ ${ }^{1}$ Trường Đại học Sư phạm tp. Hồ Chí Minh \\ ${ }^{2}$ Viện Công nghệ sinh học, Viện Hàn lâm KH \& CN Việt Nam
}

\section{TÓM TÁT}

Phân tích trình tự DNA metagenome của vi sinh vật sống trong ruột mối Coptotermes gestroi để xác định và tìm kiếm enzyme chịu được môi trường kiềm, nguồn vật liệu quan trọng để khai thác và ứng dụng trong nghiên cứu và sản xuất. Kết quả sử dụng phần mềm Alcapred để dự đoán khả năng chịu kiềm và axit của các nhóm enzyme protease, lipase, cellulase và hemicellulase từ dữ liệu metagenome của vi sinh vật trong ruột mối bao gồm: có 737 trình tự mã hóa protease chịu kiềm trong 943 trình tự và 154 trình tự mã hóa lipase chịu kiềm trong 214 trình tự từ DNA metgenome cho thấy tỷ lệ phần trăm của protease kiềm và lipase rất cao, chiếm $72 \%$ và $78 \%$. Có 338 trong tổng số 575 trình tự đã được dự đoán thuộc về nhóm enzyme chịu kiềm phân giải cellulose và hemicellulose, chiếm $59 \%$. Đây là những kết quả công bố chi tiết đầu tiên về các chuỗi gen mã hóa các enzyme chịu kiềm có nguồn gốc từ vi sinh vật sống tự do trong ruột mối của $C$. gestroi và là nguồn dữ liệu để khai thác, phân lập gen để sản xuất enzyme tái tổ hợp.

Tù khóa: Coptotermes gestroi, cellulase, enzyme chịu kiềm, hemicellulase, lipase, metagenome, protease, ruột.

Received 24 February 2016, accepted 20 September 2016 